\title{
Tecnura
}

\section{Redes inteligentes en el sistema eléctrico colombiano: Revisión de tema}

\section{Smart grids in the colombian electric system: Current situation and potential opportunities}

\author{
William Mauricio Giral Ramírez, ${ }^{1}$ Hugo José Celedón Flórez, ${ }^{2}$ Eduard Galvis Restrepo ${ }^{3}$, \\ Angela Tatiana Zona Ortiz4
}

Fecha de recepción: 12 de enero de 2017

Fecha de aceptación: 28 de abril de 2017

Cómo citar: Giral, W., Celedón, H., Galvis, E. y Zona, A. (2017). Redes inteligentes en el sistema eléctrico colombiano: revisión de tema. Revista Tecnura, 21(53), 119-137, doi: 10.14483/22487638.12396

\section{Resumen}

Contexto: el presente artículo realiza un análisis funcional de las redes eléctricas inteligentes con el propósito de establecer un marco de referencia, el cual permita identificar las principales características del sistema actual de interconexión eléctrica en Colombia. Asimismo, se presentan los incentivos propuestos por el gobierno colombiano, para apoyar proyectos de investigación y desarrollo que implementen fuentes no convencionales de energía y promuevan una gestión eficiente de ésta.

Método: con una metodología descriptiva, se presenta un modelo de arquitectura que describe la interoperabilidad de los componentes de una red inteligente.

Resultados: dentro de los resultados se establece una relación de los objetivos que en materia energética han proyectado las entidades públicas y privadas en Colombia, particularizando en las oportunidades que se tiene para dotar de inteligencia artificial el sistema eléctrico actual.

Conclusiones: desde el contexto de seguridad eléctrica, equidad social y mitigación del impacto ambiental en Colombia, el sistema energético debe afrontar los nuevos retos requeridos para satisfacer la demanda. Desde un punto de vista técnico, es necesario dotar la red tradicional con las características de una red inteligente $y$, desde una perspectiva legal, es imprescindible generar un esquema normativo y regulatorio claro que propenda por el desarroIlo de este tipo de tecnología.

Palabras clave: FNCE, red eléctrica, red inteligente, SGAM, TIC.

\begin{abstract}
Context: This paper focuses on providing a functional analysis of smart grids, with the purpose of establishing a framework to identify the main characteristics of the current electric interconnection system in Colombia. It also names the positive incentives proposed by the Colombian government to support both research and development projects that implement non-conventional energy sources and promoting energy management based on efficiency. Method: An architecture model that describes the components interoperability of a smart grid is presented using a descriptive methodology.
\end{abstract}

1 Ingeniero en Control Electrónico e Instrumentación, especialista en Bioingeniería, candidato a magister en Ingeniería Electrónica. Universidad Santo Tomás. Bogotá D.C., Colombia. Contacto: williamgiral@usantotomas.edu.co

2 Ingeniero en Electrónica, ingeniero en Mecánica, candidato a magister en Ingeniería Electrónica. Universidad Santo Tomás. Bogotá D.C., Colombia. Contacto: hugoceledon@usantotomas.edu.co

3 Ingeniero en Electrónica, magister en Ciencias de la Ingeniería, doctor en Automática, Robótica y Visión por Computador. Profesor de la Universidad Santo Tomás. Bogotá D.C., Colombia. Contacto: eduardgalvis@usantotomas.edu.co

4 Ingeniera en Telecomunicaciones, magister en Dirección y Gestión de Proyectos, doctora en Telecomunicaciones. Profesora de la Universidad Santo Tomás. Bogotá D.C., Colombia. Contacto: angelazona@usantotomas.edu.co 
Results: The results include a list of the objectives established by the Colombian public and private entities related to energy development, specially focusing on the opportunities to provide some kind of artificial intelligence to the current electrical system.

Conclusions: It is necessary for the Colombian energy system to supply the energy demand considering electrical safety, social equity, and the minimum environmental impact. These restrictions impose new challenges for the energy system itself: From a technical point of view, the traditional electrical grid must be outfitted with the characteristics of a smart grid, and from a legal perspective, it is essential to generate a clear regulatory framework that promotes the development of this type of technology.

Keywords: Smart grid, electrical grid, SGAM, ICT, FNCE.

\section{INTRODUCCIÓN}

El $80 \%$ de los gases de efecto invernadero son producidos por el consumo de recursos y energía mundial, del cual el $75 \%$ se ha concentrado en las ciudades; esto como consecuencia del significativo crecimiento de la población durante las últimas décadas, condición que da lugar a la necesidad de controlar y reducir el impacto ambiental, y garantizar calidad de vida a los habitantes. Como estrategia para dar respuesta a dichas necesidades, y en especial a los problemas energéticos relacionados a la rápida urbanización, se ha planteado la creación de ciudades inteligentes (Mohanty, Choppali, y Kougianos, 2016).

Una ciudad inteligente se puede definir como la conexión de las diferentes infraestructuras de una ciudad (física, tecnologías de la información y las comunicaciones, social y negocios), para aprovechar la inteligencia colectiva de la misma (Mohanty, et al., 2016; ITU-T Focus Group on Smart Sustainable Cities, 2015). El despliegue de una ciudad inteligente requiere un sistema eléctrico estable, confiable, seguro y de calidad ( $\mathrm{Li}, \mathrm{Xiao}, \mathrm{Gao}$, y Li, 2016), el cual incorpore en la producción energética distribuida fuentes de energía renovable, disminuyendo el uso de combustibles fósiles; como respuesta a dichos requerimientos, surge el concepto de red inteligente (RI). Las RI son, entonces, un mecanismo para la interconexión física, tecnológica y de gestión de los recursos energéticos en pro de un sistema eléctrico eficiente y confiable; de esta manera, requieren de una moderna estrategia de generación, transporte, distribución y consumo de la energía eléctrica, que se consigue mediante la integración avanzada del sensado, comunicación y control de la operación de la red eléctrica (Erol-Kantarci y Mouftah, 2015). En este contexto, las Tecnologías de la Información y las Comunicaciones (TIC) son esenciales para la comunicación entre los principales actores de la RI (agentes comercializadores, operadores de la red y clientes) y el interior del hogar del consumidor (Montes, Slootweg, y Van Eekelen, 2013).

Bajo este contexto, el gobierno colombiano creó en 2010 el programa de tecnología "Vive Digital Colombia", en su compromiso por reducir el nivel de pobreza y desempleo, aumentar la competitividad, e incentivar el uso de las TIC; el programa está alineado con los cuatro componentes del ecosistema digital: infraestructura, servicios, aplicaciones y usuarios, definidos por el "Plan Digital Colombia 2014-2018" (Ministerio de Tecnologías de la Información y las Comunicaciones, 2014), y se encuentra articulado con las áreas prioritarias definidas por el Plan Nacional Colombiano de Transformación Productiva (PTP), a saber: manufacturas, agroindustria y servicios (Ministerio de Comercio Industria y Turismo, 2014).

En este trabajo se presentan las características generales de la red eléctrica colombiana y los objetivos que en materia energética se han propuesto desde las iniciativas lideradas por la entidad pública Unidad de Planeación Minero Energética (UPME) y las entidades públicas y privadas que conforman Colombia Inteligente. Dentro del 
marco regulatorio nacional, se describen los incentivos definidos por la Ley 1715 de 2014. Se presenta el modelo de arquitectura SGAM para RI y se amplían las características de interconexión de dos de sus capas; bajo el contexto de las RI, se relacionan cuatro tecnologías que, integradas a una infraestructura TIC e internet de las cosas, Internet of things (loT, por sus siglas en inglés), facilitan la interoperabilidad de cada uno de los niveles de las RI (Congreso de la República de Colombia, 2014).

\section{DESCRIPCIÓN DE LA RED ELÉCTRICA EN COLOMBIA}

A partir de la Constitución Política de Colombia de 1991, el estado permite la participación de empresas privadas en el sector de los servicios públicos, estructurando un mercado competitivo para ofrecer a los usuarios menores tarifas y mejor servicio (Asamblea Nacional Constituyente, 1991). Este hecho dio lugar a la expedición de la Ley 142 de julio 11 de 1994 "por la cual se establece el régimen de los servicios públicos domiciliarios" y la Ley 143 de julio 12 de 1994 "por la cual se establece el régimen para la generación, interconexión, transmisión, distribución y comercialización de electricidad en el territorio nacional" (Congreso de la República de Colombia, 1994).

Una vez definidas las actividades del sector eléctrico a partir de la Ley 143, el Ministerio de Minas y Energía (MME) administra las funciones de regulación, planeación, coordinación y seguimiento (Congreso de la República de Colombia, 1994); dichas funciones son respectivamente realizadas por la Comisión de Regulación de Energía y Gas (CREG), la UPME y la Superintendencia de Servicios Públicos Domiciliarios (SSPD). Asimismo, para la planeación, supervisión y control de los recursos de generación, interconexión y transmisión del Sistema Interconectado Nacional (SIN) se cuenta con la operación integrada del Centro Nacional de Despacho (CND), los acuerdos técnicos del Administrador de Consejo Nacional de Operación (CON), y la operación de mercados del Administrador del
Sistema de Intercambios Comerciales (ASIC) dependencia del CND (Cruz, et al., 2013).

El suministro eléctrico colombiano depende del Sistema Interconectado Nacional y de los sistemas locales de las Zonas No Interconectadas (ZNI); de acuerdo al Plan Indicativo de Expansión de Cobertura de Energía Eléctrica 2013-2017, la interconexión al SIN es la principal alternativa para la prestación del servicio ZNI (UPME, 2013), este representa el 98,9\% de la generación instalada (Grupo Técnico Proyecto BID Marco de Cooperación Técnica CO-T1337, 2016b). En la figura 1, se describe la capacidad por tipo de planta generadora a septiembre de 2015 y se resalta la incorporación en la matriz energética nacional de la energía eólica y de la cogeneración, como resultado de la implementación de tecnologías limpias y optimización de procesos de combustión, respectivamente promovidas por los subprogramas del sector industrial, con el objetivo de crear metas de ahorro (UPME y BID, 2015).

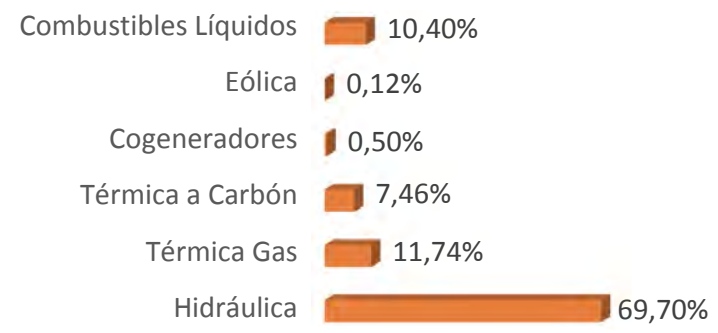

Figura 1. Capacidad instalada del SIN a septiembre de 2015

Fuente: (UPME, 2015a)

\section{Objetivos de Colombia en materia energética}

Para abordar los objetivos de Colombia en materia energética, se han tomado como referentes las propuestas presentadas por la UPME y la iniciativa Colombia Inteligente. La UPME como Unidad Administrativa Especial de Orden Nacional, ha estructurado el Plan Energético Nacional (PEN): Ideario Energético 2050, con base en un objetivo principal: lograr el abastecimiento interno y externo de 
energía y minería de manera eficiente con el mínimo impacto ambiental y generando valor para las regiones y poblaciones (UPME, 2015b).

Para llevar a cabo este objetivo general, la UPME plantea cinco objetivos específicos y dos objetivos transversales: los objetivos específicos han sido definidos dentro del contexto de la oferta energética, la demanda, la universalización, las interconexiones internacionales y la generación de valor alrededor del sector energético, para la consolidación de una Colombia productiva, eficiente, formal, competitiva y de oportunidades; articulando los objetivos específicos, se identifican los transversales, los cuales han sido propuestos atendiendo a la necesidad de contar con información, conocimiento, recurso humano, e innovación para la implementación de la energía nacional y el ordenamiento territorial, ambiental y legal (UPME, 2015b).

Por su parte, Colombia Inteligente es una iniciativa público-privada creada a finales de 2010, con el propósito de promover y desarrollar soluciones para el sector eléctrico que minimicen el impacto ambiental y optimicen el uso de la energía eléctrica (Cespedes, et al., 2012). A partir de empresas, instituciones y demás actores del sector energético colombiano, busca establecer de manera concertada lineamientos, políticas y estrategias para el desarrollo y fortalecimiento del sector. Dentro del "Informe de Gestión 2013", Colombia Inteligente propone cuatro ejes estratégicos en el marco de las RI, para el desarrollo del progreso social (Comité Directivo Colombia Inteligente, 2014). La tabla 1, describe los ejes estratégicos de Colombia Inteligente, a saber, acceso universal, seguridad y calidad, competitividad y sostenibilidad ambiental, y los relaciona con los objetivos del PEN.

La relación entre los objetivos propuestos por la UPME y las estrategias de Colombia Inteligente, refleja el interés de los agentes activos del sector energético de carácter público y privado, frente a la investigación, el desarrollo e innovación en RI.

En este contexto y bajo el marco de la Ley 1715 de 2014, el gobierno colombiano ha establecido un conjunto de incentivos para promover un servicio eléctrico continuo, de calidad, con mínimo impacto ambiental y que contribuya al desarrollo social y económico del país.

\section{Incentivos en materia energética de la Ley 1715 de 2014}

El Congreso de Colombia expidió el 13 de mayo de 2014 la Ley 1715, el propósito de esta ley es establecer un marco normativo y regulatorio que propenda por el desarrollo económico sostenible, la reducción de gases de efecto invernadero, la seguridad en el abastecimiento energético y la gestión eficiente de la energía, a través de la integración de las fuentes no convencionales de energía (FNCE) al sistema energético nacional, principalmente aqueIlas de carácter renovable (Congreso de la República de Colombia, 2014).

Asimismo, el MME a través del Decreto 2143 del 4 de noviembre de 2015 adiciona un nuevo título al Decreto 1073 de 2015, para el desarrollo de los incentivos propuestos por la Ley 1715. De esta manera, los interesados en realizar inversiones en proyectos de investigación y desarrollo en FNCE y gestión eficiente de la energía, podrán acceder a incentivos tributarios, arancelarios o contables (Ministerio de Minas y Energía, 2015a), (Ministerio de Minas y Energía, 2015b), (Correa, Maruland y Panesso, 2016).

Simultáneo a los incentivos planteados, es imprescindible definir un modelo de arquitectura para RI que, articulado con el marco normativo y regulatorio, permita identificar las funciones e interoperabilidad de cada uno de los actores que integran el sistema eléctrico. El presente trabajo realiza un análisis de la tendencia de las RI en Colombia bajo el modelo de arquitectura, Smart Grid Architecture Model (SGAM, por sus siglas en inglés).

\section{MODELO DE ARQUITECTURA SGAM PARA REDES INTELIGENTES}

El modelo de arquitectura SGAM descrito en la figura 2 y referido durante el desarrollo de este 
Tabla 1. Relación de los ejes estratégicos Colombia Inteligente con los objetivos del PEN

\begin{tabular}{|c|c|c|c|c|}
\hline \multicolumn{5}{|c|}{$\begin{array}{l}\text { Objetivos específicos del pen. } \\
\text { Colombia un país }\end{array}$} \\
\hline 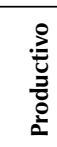 & 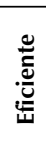 & 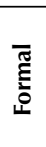 & 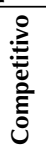 & ¿ \\
\hline
\end{tabular}

\section{Acceso universal}

Proveer electricidad a todos los colombianos, promover el desarrollo económico y social, implementar RI en ZNI y emplear microredes en las ZNI.

\section{Seguridad y calidad}

Promover la implementación de RI, consolidar una canasta energética acorde a los recursos del país, minimizar el riesgo de períodos de escasez, proveer electricidad de calidad y alta confiabilidad y potencializar las exportaciones de energía.

\section{Competitividad}

Implementar RI para una mayor eficiencia y menores costos, atraer industrias electro intensivas, proporcionar energía a un atractivo precio, flexibilizar la incorporación de nuevas fuentes de energía renovable, reducir las pérdidas técnicas y no-técnicas, incentivar procesos para la gestión de la demanda, promover una cultura de uso eficiente de la electricidad, incentivar el desarrollo e integración de tecnologías en RI, adquirir experiencia en los procesos de recolección de datos, procesamiento de información e integración de aplicaciones.

\section{Sostenibilidad ambiental}

Reducir el impacto ambiental, minimizar el impacto por efecto de los proyectos de generación, disminuir huellas de carbono e impulsar la penetración de la movilidad eléctrica.

OBSERVACIÓN: Las marcas indican una mayor relación mientras las áreas en blanco indican una menor relación.

Fuente: (Comité Directivo Colombia Inteligente, 2014; UPME, 2015b)

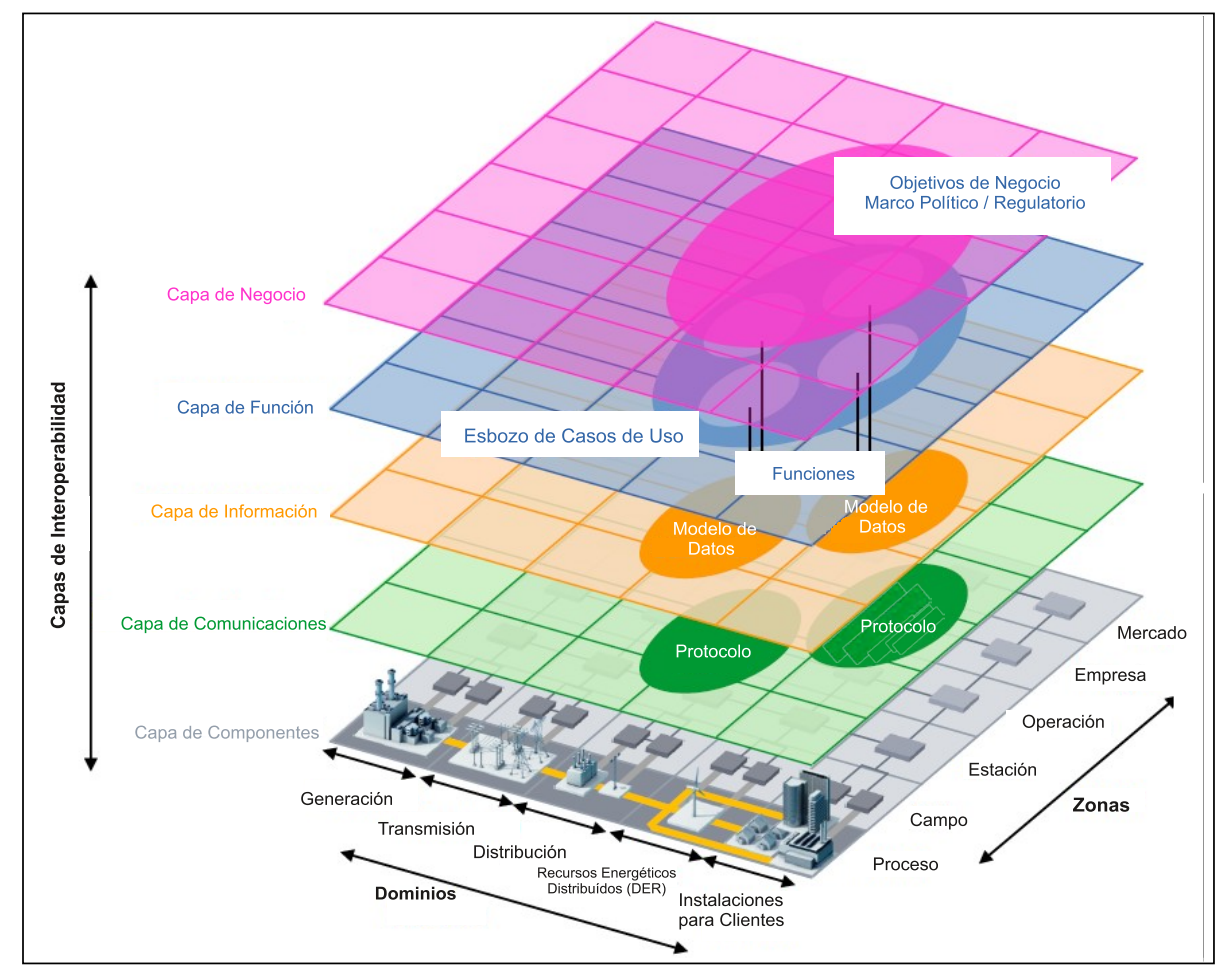

Figura 2. Modelo de referencia SGAM

Fuente: (CEN-CENELEC-ETSI Smart Grid Coordination Group, 2014; Neureiter, et al.) 
trabajo como SGAM, se emplea para analizar y visualizar las RI desde una posición tecnológica neutral. Mediante los principios de universalidad, localización, flexibilidad e interoperabilidad, SGAM proporciona un enfoque que hace frente a la complejidad de las RI, permitiendo la transición desde el estado actual de la red eléctrica hacia los futuros escenarios de las RI (CEN-CENELEC-ETSI Smart Grid Coordination Group, 2014; Neureiter, Uslar, Engel y Lastro, s.f.).

SGAM está conformado por dominios, zonas e interoperabilidad, subdivididos a su vez en niveles y capas como se describe en la tabla 2; los dominios físicos de la red corresponden a la cadena completa de conversión de la energía eléctrica: generación, transmisión, distribución, recursos energéticos distribuidos, Distributed Energy Resources (DER, por sus siglas en inglés), e instalaciones para los usuarios. Las zonas definen los niveles jerárquicos involucrados en la gestión del sistema de energía eléctrica: proceso, campo, estación, operación, empresa y mercado. Mientras que la relación entre las zonas y los dominios, se establece mediante las capas de interoperabilidad: componente, comunicación, información, función y negocio (CEN-CENELEC-ETSI Smart Grid Coordination Group, 2014).

En el Plan de Expansión de Referencia Generación-Transmisión 2015-2029 (UPME, 2015a), se realiza un análisis y se proponen diversas estrategias en cuatro de los dominios propuestos por SGAM: generación, transmisión, distribución y recursos energéticos distribuidos; de manera que se plantean los requerimientos técnicos del sistema de energía eléctrica de Colombia dentro de la proyección de demanda para el desarrollo económico del país en diferentes escenarios temporales.

Como caso particular dentro del Plan Energético Nacional (PEN), y bajo el marco normativo y regulatorio de la ley 1715 de 2014 (Congreso de la República de Colombia, 2014), se establece la necesidad de integrar en la canasta de generación eléctrica fuentes no convencionales (FNCE), principalmente aquellas de carácter renovable (UPME, 2015b). De esta manera, a partir de la reunión de divulgación del 15 de noviembre de 2016, la UPME a través del Plan de Expansión de Referencia Generación-Transmisión 2016-2030 (UPME, 2016), en etapa de aprobación por el Ministerio de Minas y Energía (MME), presenta los escenarios a largo plazo, tomando como caso base la actualización de la estrategia doce del Plan de Expansión de Referencia Generación-Transmisión 2015-2029, la cual se sustenta en 1200 MW de la segunda fase de Ituango, $227 \mathrm{MW}$ de generación hidráulica, $147 \mathrm{MW}$ de plantas térmicas, 1456 MW eólicos y 569,2 MW con FNCE (UPME, 2015a).

A nivel mundial, según el índice del trilema energético de 2015 (Energy Trilemma Index, 2015) del consejo mundial de energía (WEC, World Energy Council), Colombia ocupó para el 2015 el puesto dieciocho de 130 países analizados, cayendo dos puestos con respecto al 2014, donde ocupó el puesto dieciséis de 129 países (World Energy Council, 2015). Este índice evalúa tres criterios: seguridad de suministro energético, equidad energética y sostenibilidad ambiental. Clasificando a los países conforme a dicha evaluación y particularizando los resultados de 2015, Colombia se encuentra en el puesto trece a nivel de seguridad de suministro energético, en el puesto 58 de equidad energética y en el puesto tres de sostenibilidad ambiental; el resultado alentador en la seguridad de suministro energético y sostenibilidad energética, es un indicador del crecimiento de consumo en relación con el crecimiento del PIB (producto interno bruto), como resultado de contar en el país con grandes reservas de petróleo conjugadas con el alto compromiso político y económico (World Energy Council, 2015).

La evaluación más crítica es la equidad energética, por lo que Colombia debe promover la ampliación del sistema de energía eléctrica con el fin de aumentar la cobertura; esta ampliación evidencia un área de oportunidad para la implementación de 
Tabla 2. Descripción de componentes que integran SGAM

\begin{tabular}{|c|c|c|c|}
\hline & Com & nente & Descripción \\
\hline \multirow{5}{*}{ Dominio } & \multirow{5}{*}{ Nivel } & Generación & $\begin{array}{l}\text { Generación de energía eléctrica a partir de combustibles fósiles, plantas hi- } \\
\text { droeléctricas, plantas de energía nuclear, parques eólicos, plantas de energía solar } \\
\text { fotovoltaica, usualmente conectadas al sistema de transmisión. }\end{array}$ \\
\hline & & Transmisión & Infraestructura y organización para transportar electricidad a amplias distancias. \\
\hline & & Distribución & Infraestructura y organización requerida para distribuir electricidad a usuarios. \\
\hline & & $\begin{array}{l}\text { Recursos energéti- } \\
\text { cos distribuidos }\end{array}$ & $\begin{array}{l}\text { Recursos directamente conectados a la red de distribución pública, aplicando } \\
\text { tecnologías para la generación de energía a pequeña escala (rango de } 3 \mathrm{~kW} \text { a } 10 \\
\mathrm{MW} \text { ). }\end{array}$ \\
\hline & & $\begin{array}{l}\text { Instalaciones para } \\
\text { usuarios }\end{array}$ & $\begin{array}{l}\text { Usuarios y productores de electricidad. Incluye instalaciones de tipo industrial, } \\
\text { comercial y para el hogar. }\end{array}$ \\
\hline \multirow{6}{*}{ Zona } & \multirow{6}{*}{ Nivel } & Mercado & Comercio de energía. \\
\hline & & Empresa & $\begin{array}{l}\text { Procesos comerciales, organizacionales y de servicios e infraestructuras para } \\
\text { empresas. }\end{array}$ \\
\hline & & Operación & $\begin{array}{l}\text { Operación de los sistemas de: gestión de distribución, gestión energética, gestión } \\
\text { de plantas virtuales, a través de la agrupación de DER y sistemas de gestión de } \\
\text { carga para flotas de vehículos eléctricos ( } E V-E l e c t r i c \text { Vehicle). }\end{array}$ \\
\hline & & Estación & $\begin{array}{l}\text { Datos del nivel campo para concentrar datos, agregar funcionalidades, automati- } \\
\text { zar subestaciones, implementar sistemas locales de supervisión y realizar control } \\
\text { y adquisición de datos (SCADA - Supervisory Control And Data Acquisition). }\end{array}$ \\
\hline & & Campo & Equipos para protección, control y monitoreo del nivel proceso. \\
\hline & & Proceso & $\begin{array}{l}\text { Transformación física y química de energía y de los equipos directamente involu- } \\
\text { crados. }\end{array}$ \\
\hline \multirow{5}{*}{$\begin{array}{l}\text { Interope- } \\
\text { rabilidad }\end{array}$} & \multirow{5}{*}{ Capa } & Negocio & $\begin{array}{l}\text { Marco regulatorio, estructuras económicas y políticas, modelos de negocio, por- } \\
\text { tafolio de negocios de mercados involucrados, capacidad de negocio y proyectos } \\
\text { de negocio específico. }\end{array}$ \\
\hline & & Función & Representa la actividad de las empresas del sistema energético. \\
\hline & & Información & $\begin{array}{l}\text { Tipo de información intercambiada entre funciones, servicios y componentes, } \\
\text { incluyendo el registro y almacenamiento de datos. }\end{array}$ \\
\hline & & Comunicación & Protocolos y mecanismos para el intercambio de información entre componentes. \\
\hline & & Componente & $\begin{array}{l}\text { Nivel físico del sistema eléctrico que incluye dispositivos, aplicaciones y equipos } \\
\text { de protección, telecontrol e infraestructura asociada a redes y computadores. }\end{array}$ \\
\hline
\end{tabular}

Fuente: (CEN-CENELEC-ETSI Smart Grid Coordination Group, 2012)

RI en Colombia, basada en energías no convencionales, la mejora de la calidad y la fiabilidad de los servicios de energía, la diversificación de la matriz energética, y el sostenimiento del desarrollo económico sin aumentar las emisiones de dióxido de carbono (World Energy Council, 2015).

\section{Red inteligente}

Una RI busca satisfacer la demanda de los usuarios finales a través de tecnologías digitales, que permiten un continuo monitoreo y control sobre el transporte de la energía eléctrica desde las fuentes de generación; de esta manera, las RI permiten que el sistema sea controlado eficientemente por las partes interesadas. A saber, generador, operador de red, usuario final y mercados relacionados, con el objetivo de minimizar los costos e impacto medio ambiental (Wei, Zhou y Zhu, 2009), mientras se incrementa la confiabilidad, la resiliencia y la estabilidad del sistema (International Energy Agency, 2011). La tabla 3 realiza la comparación de las características técnicas de la red eléctrica actual colombiana frente a una RI. 
Tabla 3. Comparación técnica de la red eléctrica actual colombiana frente a una RI

\begin{tabular}{ll}
\hline \multicolumn{1}{c}{$\begin{array}{c}\text { Red eléctrica actual } \\
\text { colombiana }\end{array}$} & \multicolumn{1}{c}{ Red inteligente } \\
\hline Electromecánica & Digital \\
\hline Comunicación unidireccional & Comunicación bidireccional \\
\hline Generación centralizada & Generación distribuida \\
\hline Pocos sensores & Sensores a lo largo de la RI \\
\hline Monitoreo manual & Auto monitoreo \\
\hline Restauración manual & Reconfiguración automática \\
\hline Fallas y apagones & Adaptativo y aislado \\
\hline Control limitado & Control generalizado \\
\hline Pocas opciones de imple- & Múltiples opciones de \\
mentación de TIC para los & implementación de TIC para \\
clientes & los clientes \\
\hline
\end{tabular}

Fuente: (Fang, Misra, Xue y Yang, 2012)

A pesar de las debilidades técnicas que presenta el actual sistema energético colombiano para el desarrollo de las RI, hay una gran oportunidad desde el marco normativo y regulatorio, que mediante incentivos económicos promueve la integración de fuentes no convencionales de energía renovable (FNCER) a la red tradicional (Congreso de la República de Colombia, 2014) tendientes a la generación distribuida, a la eficiencia energética e inclusión de las ZNI para el desarrollo económico sostenible del país.

Desde esta perspectiva se hace imprescindible dotar a la red tradicional con características propias de las $\mathrm{RI}$, a través de la integración de cuatro tecnologías: infraestructura de medida avanzada, Advanced Metering Infrastructure (AMI, por sus siglas en inglés), automatización avanzada de la red de distribución, Advanced Distribution Automation (ADA, por sus siglas en inglés ), DER y vehículos eléctricos, Electric Vehicle (EV, por sus siglas en inglés). Las debilidades, oportunidades, fortalezas y amenazas de la incorporación de estas tecnologías en Colombia se presentan en Grupo Técnico Proyecto BID Marco de Cooperación Técnica CO-T1337 (2016a; 2016b; 2016c). En la siguiente sección se explican las características de las tecnologías AMI, ADA, DER y EV, cuyo desarrollo e implementación contribuirían con los objetivos definidos por la UPME (UPME, 2015b) y Colombia Inteligente (Comité Directivo Colombia Inteligente, 2014).

\section{Infraestructura de medida avanzada (AMI)}

AMI es una tecnología que integra contadores inteligentes, Smart Meter (SM, por sus siglas en inglés), redes de comunicación y sistemas para la administración de datos. Mediante una comunicación de tipo bidireccional entre el SM y las empresas prestadoras del servicio de energía eléctrica, AMI tiene como objetivo gestionar aplicaciones para hacer uso eficiente de la energía, tendientes a la reducción del impacto ambiental (Ozgur, Tonyali, Akkaya y Senel, 2016; Gopi y Lalu, 2016; Yu, et al., 2014; Rashed, Fung, Mohammadi y Raahemifar, 2014).

La principal aplicación de AMI para los usuarios es la disposición de información de consumo. Para las empresas prestadoras del servicio de energía eléctrica, donde se requiere un mayor control, la tecnología AMI permite realizar: medición de la red, operación remota, limitación de potencia, medida de generación distribuida, detección de manipulación, fijación de precio basada en el tiempo, limitantes de carga para propósitos de respuesta a la demanda, monitoreo de la calidad energética y notificación de corte y falla (Rashed, et al., 2014; Kuzlu, Pipattanasomporn y Rahman, 2014).

Frente a la instalación de contadores inteligentes en Colombia, se requiere la definición de un marco regulatorio y de beneficios para los usuarios que viabilice socialmente la implementación de este tipo de tecnología (Grupo Técnico Proyecto BID Marco de Cooperación Técnica CO-T1337, 2016b). Dentro de los proyectos piloto, la empresa de servicios públicos EMCALI, ha instalado alrededor de 18000 medidores AMI, como resultado de este proceso, EMCALI ha obtenido: una reducción de las emisiones de $\mathrm{CO}_{2}$, un ahorro económico 
para los clientes y un aumento de personal calificado (Caicedo, Castillo, Morales, Echeverry y García, 2015).

\section{Automatización avanzada de la red de distribución (ADA)}

Las tecnologías para ADA permiten que el sistema de distribución de energía eléctrica sea completamente controlable y flexible, para aumentar la eficiencia, sostenibilidad y confiabilidad (Elkhatib, Shatshat y Salama, 2012; Quang-Dung, Chao, Chon-Wang, Derakhshani, Mahsa, Le-Ngoc, 2014).

Algunas aplicaciones para ADA son: supervisión, control y adquisición de datos (SCADA; Supervisory Control And Data Acquisition), control de voltaje y potencia reactiva, localización de fallas, reconfiguración automática, aislamiento y servicio de restauración, información al usuario, tarificación horaria, gestión de activos y telemando (Zavoda, 2010). En Grupo Técnico Proyecto BID Marco de Cooperación Técnica COT1337, (2016b) se plantea el desarrollo gradual de estas tecnologías sobre la red tradicional colombiana, particularmente en las redes urbanas, a través del uso de interruptores y seccionadores con telecontrol.

\section{Recursos energéticos distribuidos (DER)}

El interés por reducir la emisión de gases de efecto invernadero y disminuir el costo asociado al consumo eléctrico, ha aumentado las oportunidades de los DER en las RI (Madani, et al., 2015; W. Wang, Xu y Khanna, 2011). El objetivo de esta tecnología es optimizar la operación y planeamiento de la red de distribución eléctrica (Carvajal y David, 2013). Para este propósito previo a la implementación de un sistema de generación distribuida, Distributed Generation (DG, por sus siglas en inglés), los DER requieren de una etapa de planeación, donde se identifican las zonas geográficas de mayor impacto social y económico, y se determina el modelo y método de instalación (Georgilakis, Member y Hatziargyriou, 2013).

Los trabajos que se han llevado a cabo en torno a las DER, cubren dos funcionalidades: la primera corresponde a sistemas de microgeneración con conexión y control de corriente alterna Alternating Current (AC, por sus siglas en inglés) y corriente directa, Direct Current (DC, por sus siglas en inglés), ampliadas en Vasquez, Guerrero, Miret, Castilla y Vicuña (2010), Justo, Mwasilu, Lee y Jung (2013), Marzband, Parhizi, Savaghebi y Guerrero (2016), Guerrero, Chandorkar, Lee y Loh (2013), Guerrero, Loh, Lee y Chandorkar (2013), Wang, Guerrero, Chen y Blaabjerg (2010) y Rahman, et al., (2015). La segunda está relacionada con los sistemas para el almacenamiento de energía, abordados en Dimitrov, Piroddi y Prandini (2016).

Los alcances de los sistemas de microgeneración y almacenamiento de energía incluyen los siguientes procesos de optimización: minimización de pérdidas totales del sistema, minimización del índice de duración promedio de la interrupción, System Average Interruption Duration Index (SAIDI, por sus siglas en inglés), minimización del costo (Braslavsky, Wall y Reedman, 2015), maximización de la capacidad de DG y maximización de relación beneficio-costo.

En Colombia, gracias a la disponibilidad de recursos naturales como el agua, la radiación solar, y el viento, aunado a la capacidad de aprovechar materia orgánica a través de la biomasa, se tiene una gran oportunidad para abastecer las ZNI con FNCER dentro de los DER (UPME y BID, 2015); esta tendencia hacia la implementación de nuevas soluciones energéticas para las ZNI fortalece el desarrollo sostenible social, económico y ambiental.

\section{VEHÍCULOS ELÉCTRICOS (EV)}

El desarrollo del transporte eléctrico ha reducido la emisión de gases de efecto invernadero como resultado de la disminución del uso de combustibles fósiles (Chen, Tan y Quek, 2014); simultáneo a este 
hecho, la integración a las RI de los EV, mediante la incorporación de las TIC, han permitido nuevas estrategias para el control energético, donde los usuarios de los EV participan de manera activa en el mercado eléctrico (Congreso de la República de Colombia, 2014). Estas estrategias, son Ilevadas a cabo durante el proceso de carga de los EV, como resultado se obtiene el aplanamiento de picos generados cuando hay alta demanda, transporte de más energía eléctrica sin incrementar la capacidad de la red, desplazamiento de cargas a horas de menor demanda, regulación de la frecuencia y balance de la energía eléctrica en tiempo real (Veldman y Verzijlbergh, 2015).

Actualmente son utilizados dos tipos de interacciones entre los EV y la red de energía eléctrica: red al vehículo, Grid to Vehicle (G2V, por sus siglas en inglés) y vehículo a la red, Vehicle to Grid (V2G, por sus siglas en inglés). En la interacción G2V, la batería de un EV puede ser cargada de forma unidireccional desde una fuente externa; en V2G, el flujo energético es bidireccional, desde la red hacia el EV mientras está en la etapa de carga y desde el EV hacia la red cuando está en la etapa de descarga (Mukherjee y Gupta, 2015). Nuevos modos de operación como casa a vehículo, Home to Vehicle ( $\mathrm{H} 2 \mathrm{~V}$, por sus siglas en inglés) y vehículo para red, Vehicle for Grid (V4G, por sus siglas en inglés), se pueden ver en Monteiro, Pinto y Afonso (2016).

Debido a la importancia del tipo de acumuladores empleados por los EV, en Xing, et al. (2016), se presentan algunas características asociadas a las baterías empleadas y se propone un sistema de almacenamiento de energía magnética por superconducción para lograr estabilidad del sistema de distribución de la RI. Un modelo del comportamiento de movilidad y demanda para EV es ampliado en Kennel, Gorges y Liu (2013), donde las baterías de EV en este caso son empleadas dentro de una RI para el control de frecuencia de la carga.

El gobierno colombiano, desde el marco normativo y regulatorio, ha promovido la ejecución de proyectos de solución en materia de transporte para el desarrollo de una movilidad sostenible
(Dangond, Jolly, Monteoliva y Rojas, 2011). Quintana (2014) propone un modelo de masificación de vehículos eléctricos en la ciudad de Bogotá D.C., para el periodo comprendido entre 2014 y 2034; bajo el análisis de tres escenarios, establece la necesidad de ampliar y modernizar la red eléctrica para garantizar puntos de recarga en la ciudad y propone una gestión de demanda en tiempo real, tendiente a la incorporación de redes inteligentes en los sistemas de distribución.

\section{TECNOLOGÍAS DE LA INFORMACIÓN Y DE LAS COMUNICACIONES}

La interoperabilidad entre las TIC y la capa de componentes de SGAM, permite la integración avanzada del sensado, las comunicaciones y el control, en la cadena completa de conversión de energía eléctrica (ITU-T Focus Group on Smart Sustainable Cities, 2014). Desde la perspectiva de las TIC y con el propósito de establecer un mapa de ruta para la estandarización de actividades asociadas a las RI, la Unión Internacional de Telecomunicaciones, The International Telecommunication Union (ITU, por sus siglas en inglés), a partir del Sector para la Estandarización de Telecomunicaciones ITU-T, creó en Febrero de 2010 FG-Smart (Focus Group on Smart Grid; Lee y Su, 2013; ITU-T Focus Group on Smart Sustainable Cities, 2014).

FG-Smart, bajo un modelo conceptual propuesto por el Instituto Nacional de Estándares y Tecnología, Institute of Standards and Technology (NIST, por sus siglas en inglés), describe una arquitectura de RI formada por cinco dominios correspondientes a: red eléctrica, medición inteligente, cliente, red de comunicación y proveedor de servicios (CEN-CENELEC-ETSI Smart Grid Coordination Group, 2014; Lee y Su, 2013; Budka, Deshpande y Thottan, 2014; Nieße, Tröschel y Sonnenschein, 2014). La figura 3, identifica las áreas que relacionan los dominios del modelo conceptual FG-Smart con SGAM.

El modelo FG-Smart para RI, está conformado por los dominios red eléctrica, medición inteligente, cliente, red de comunicación y proveedor 


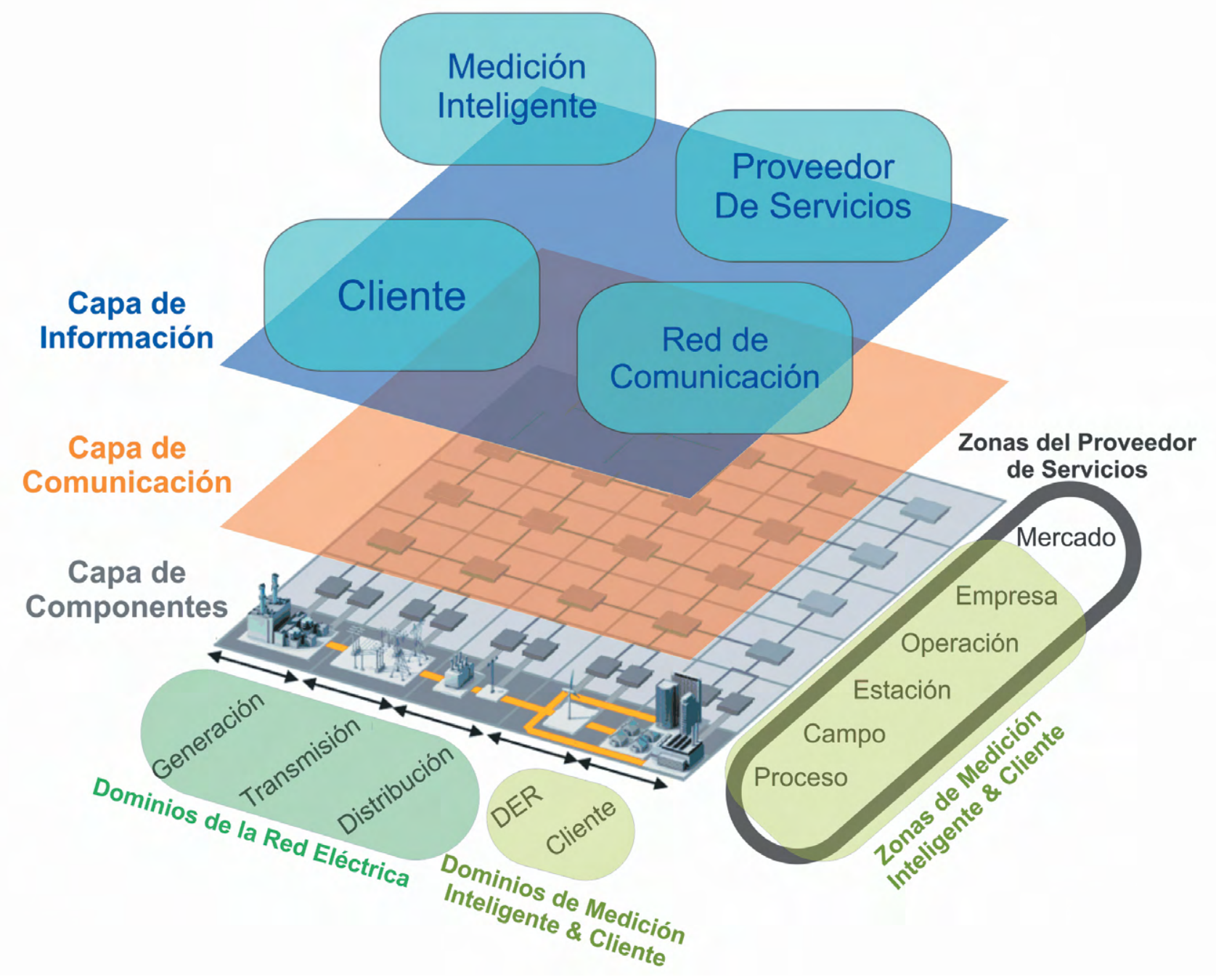

Figura 3. Relación dominios del modelo conceptual FG-Smart y la capa de componentes de SGAM

Fuente: (CEN-CENELEC-ETSI Smart Grid Coordination Group, 2014; Lee y Su, 2013; Budka, et al., 2014; Nieße, et al., 2014)

de servicios. Mediante el dominio red de comunicación y capa de comunicación, los dispositivos para supervisión y control de la cadena completa de conversión de energía, integrados en el dominio red eléctrica, intercambian información con el proveedor de servicios; por su parte, para la medición inteligente FG-Smart, correspondiente a los dominios DER e instalaciones para clientes SGAM, se recurre a las capas de información y comunicación para habilitar la integración y participación activa del dominio cliente (CEN-CENELEC-ETSI Smart Grid Coordination Group, 2014; Lee y Su, 2013; Nieße, et al., 2014).
Asimismo, el modelo conceptual FG-Smart puede ser definido desde los dominios, zonas y capas de componentes, de comunicación y de información de SGAM; la capa de comunicación, como se observa en la Figura 4, presenta la relación entre los dominios y las zonas para la red de área doméstica, Home Area Network (HAN, por sus siglas en inglés), red de área de vecindario, Neighborhood Area Network (NAN, por sus siglas en inglés) y red de área global Wide Area Network, (WAN, por sus siglas en inglés).

Como se ve en la figura 4, FG-Smart aplica la red HAN para la interoperabilidad de los dominios 

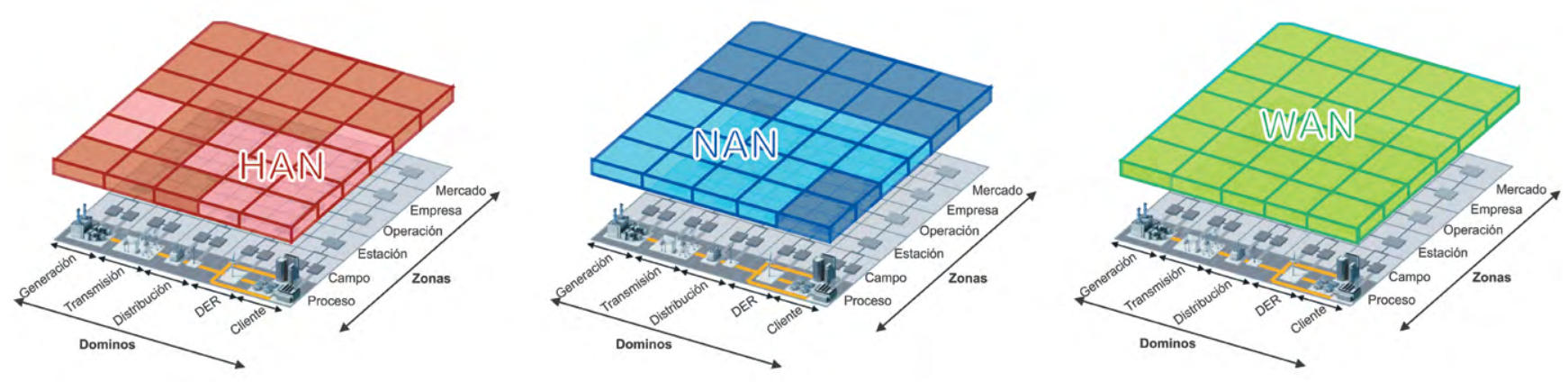

Figura 4. Área de aplicación de las redes HAN, NAN, WAN en SGAM.

Fuente: (CEN-CENELEC-ETSI Smart Grid Coordination Group, 2014; W. Li y Zhang, 2014; Lee y Su, 2013)

generación y distribución con las zonas campo y estación, el dominio DER con las zonas proceso, campo y estación, y el dominio cliente con las zonas proceso, campo, estación y operación. Asimismo, aplica la red NAN para la interoperabilidad de los dominios generación, transmisión con las zonas proceso, campo y estación, los dominios distribución, DER con las zonas proceso, campo, estación y operación, y el dominio cliente con las zonas estación y operación; finalmente, FG-Smart aplica la red WAN para la interoperabilidad de todos los dominios y zonas. Las características técnicas de las redes HAN, NAN y WAN se presentan en la tabla 4.

Tabla 4. Descripción de redes HAN, NAN, WAN

\begin{tabular}{|c|c|c|c|c|}
\hline Descripción & $\begin{array}{l}\text { Velocidad } \\
\text { de datos }\end{array}$ & Cobertura & $\begin{array}{l}\text { Implementada sobre } \\
\text { tecnologías }\end{array}$ & Extensiones \\
\hline \multicolumn{5}{|l|}{ Red HAN } \\
\hline $\begin{array}{l}\text { Localizado en el dominio del cliente en } \\
\text { el hogar se extiende hacia aplicaciones } \\
\text { AMI. Permite a los clientes conocer el } \\
\text { costo del uso de la energía eléctrica, el } \\
\text { comportamiento de su consumo y tomar } \\
\text { parte de las aplicaciones inteligentes. }\end{array}$ & $\begin{array}{l}\text { Superior a } \\
100 \mathrm{kbps}\end{array}$ & $\begin{array}{l}\text { Superior a } \\
100 \mathrm{~m}\end{array}$ & $\begin{array}{l}\text { ZigBee, WiFi, HomePlug } \\
\text { o PLC, Z-wave, Bluetoo- } \\
\text { th, Ethernet, M-Bus. }\end{array}$ & $\begin{array}{l}\text { Red de Área de Edificio } \\
\text { (BAN - Building Area Ne- } \\
\text { twork) para aplicaciones de } \\
\text { negocio. } \\
\text { Red de Área Industrial (IAN } \\
\text { - Industrial Area Network) } \\
\text { para ajuste industrial. }\end{array}$ \\
\hline \multicolumn{5}{|l|}{ Red NAN } \\
\hline $\begin{array}{l}\text { Red de comunicación para áreas energé- } \\
\text { ticas de distribución, la cual actúa como } \\
\text { puente entre el cliente y la subestación a } \\
\text { través de colectores, puntos de acceso y } \\
\text { concentradores de datos. }\end{array}$ & $\begin{array}{l}100 \mathrm{kbps} \text { a } \\
10 \mathrm{Mbps}\end{array}$ & $\begin{array}{l}\text { Superior a } \\
10 \mathrm{~km}\end{array}$ & $\begin{array}{l}\text { Red en malla ZigBee, } \\
\text { Red en malla WiFi, PLC, } \\
\text { WiMAX, Celular, DSL, } \\
\text { Ethernet, Fibra óptica, } \\
\text { Cable Coaxial. }\end{array}$ & $\begin{array}{l}\text { Red de Área de Campo } \\
\text { (FAN - Field Area Network) }\end{array}$ \\
\hline \multicolumn{5}{|l|}{ Red WAN } \\
\hline $\begin{array}{l}\text { Proporciona comunicación entre las } \\
\text { empresas de servicio eléctrico y las sub- } \\
\text { estaciones. Son empleadas para monito- } \\
\text { reo y protección. }\end{array}$ & $\begin{array}{l}10 \text { Mbps a } 1 \\
\text { Gbps }\end{array}$ & $\begin{array}{l}\text { Superior a } \\
100 \mathrm{~km}\end{array}$ & $\begin{array}{l}\text { Óptica, Celular, WiMAX, } \\
\text { Satelital. }\end{array}$ & \\
\hline
\end{tabular}

Fuente: (Khan, Rehmani y Reisslein, 2016; Emmanuel y Rayudu, 2016; Li y Zhang, 2014; Kuzlu, et al., 2014; CEN-CENELEC-ETSI Smart Grid Coordination Group, 2014) 
Aunado a la selección de la red de acuerdo a los requerimientos de la aplicación de las RI, es determinante la selección del tipo de medio de transmisión. Las características técnicas de los medios de transmisión guiados y no guiados empleados en RI se presentan en la tabla 5.

El Ministerio de Tecnologías de la Información y las Comunicaciones de Colombia, mediante la Resolución 1564 del 25 de agosto de 2016, actualizó el Plan Vive Digital 2014-2018, dando continuidad al Plan Vive Digital 2010-2014, el cual en materia de infraestructura de telecomunicaciones logró: cobertura de redes de fibra óptica en $96 \%$ de los municipios del país, seis operadores en 4G, nueve cables submarinos y en todos los centros poblados rurales por lo menos un punto de acceso a internet por cada 100 habitantes. A partir de estos resultados, el Plan Vive Digital 2014-2018, ha propuesto como meta para el 2018: aumentar a 27 millones las conexiones a internet de banda ancha (valor tres veces mayor al alcanzado en el año 2014), cobertura de servicios móviles $4 G$ a todos

Tabla 5. Medios de transmisión para RI

\begin{tabular}{|c|c|c|c|c|}
\hline \multicolumn{2}{|r|}{ Medio de transmisión } & \multirow{2}{*}{$\begin{array}{l}\text { Velocidad de datos } \\
\text { PON: } 155 \mathrm{Mbps} \text { a } 2.5 \mathrm{Gbps} \\
\text { WDM: } 40 \mathrm{Gbps} \\
\text { SONET/SDH: } 10 \mathrm{Gbps}\end{array}$} & \multirow{2}{*}{$\begin{array}{l}\text { Cobertura } \\
\text { Superior a } 60 \mathrm{~km} \\
\text { Superior a } 100 \mathrm{~km} \\
\text { Superior a } 100 \mathrm{~km} \\
\end{array}$} & \multirow{2}{*}{$\begin{array}{l}\text { Aplicaciones } \\
\text { NAN, WAN }\end{array}$} \\
\hline \multirow{5}{*}{ Guiado } & Fibra Óptica & & & \\
\hline & $\begin{array}{l}\text { DSL (del inglés, Digital Subs- } \\
\text { criber Line) }\end{array}$ & $\begin{array}{l}\text { ADSL: } 1 \text { a } 8 \text { Mbps } \\
\text { HDSL: } 2 \text { Mbps } \\
\text { VDSL: } 15 \text { a } 100 \text { Mbps }\end{array}$ & $\begin{array}{l}\text { Superior a } 5 \mathrm{~km} \\
\text { Superior a } 3.6 \mathrm{~km} \\
\text { Superior a } 1.5 \mathrm{~km} \\
\end{array}$ & $\begin{array}{l}\text { HAN, NAN, WAN } \\
\text { AMI }\end{array}$ \\
\hline & Cable Coaxial & DOCSIS: $172 \mathrm{Mbps}$ & Superior a $28 \mathrm{~km}$ & NAN \\
\hline & PLC & $\begin{array}{l}\text { HomePlug: } 14 \text { a } 200 \mathrm{Mbps} \\
\text { Narrowband: } 10 \text { a } 500 \mathrm{kbps}\end{array}$ & $\begin{array}{l}\text { Superior a } 200 \mathrm{~m} \\
\text { Superior a } 3 \mathrm{~km}\end{array}$ & $\begin{array}{l}\text { HAN, NAN } \\
\text { AMI }\end{array}$ \\
\hline & Ethernet & 802.3x: 10 Mbps a 10 Gbps & Superior a $100 \mathrm{~m}$ & HAN, NAN \\
\hline \multirow{13}{*}{ No guiado } & Z-Wave & 40 kbps & $\begin{array}{l}30 \mathrm{~m} \text { al interior } \\
100 \mathrm{~m} \text { al aire libre }\end{array}$ & HAN \\
\hline & 6LoWPAN & $40 \mathrm{kbps}$ & Superior a $200 \mathrm{~m}$ & HAN \\
\hline & Bluetooth 802.15 .1 & Máximo 1 Mbps & $10 \mathrm{~m}$ usualmente & $\begin{array}{l}\text { HAN } \\
\text { AMI }\end{array}$ \\
\hline & ZigBee Estándar 802.15.4 & $250 \mathrm{kbps}$ & $\begin{array}{l}10 \mathrm{~m} \text { a } 75 \mathrm{~m} \text { punto a } \\
\text { punto }\end{array}$ & \multirow{3}{*}{$\begin{array}{l}\text { HAN, NAN } \\
\text { AMI }\end{array}$} \\
\hline & ZigBee Pro & $250 \mathrm{kbps}$ & Superior a $1600 \mathrm{~m}$ & \\
\hline & WiFi $802.11 x$ & 2 a $600 \mathrm{Mbps}$ & Superior a $250 \mathrm{~m}$ & \\
\hline & WiMAX 802.16 & $72 \mathrm{Mbps}$ & $9 \mathrm{~km}$ & $\begin{array}{l}\text { NAN, WAN } \\
\text { AMI, ADA }\end{array}$ \\
\hline & Celular 2G-GSM & $14 \mathrm{kbps}$ & $10 \mathrm{~km}$ & \multirow{2}{*}{$\begin{array}{l}\text { NAN, WAN, } \\
\text { AMI, EV, ADA }\end{array}$} \\
\hline & Celular 2.5G-GPRS & $171 \mathrm{kbps}$ & $10 \mathrm{~km}$ & \\
\hline & $\begin{array}{l}\text { Celular 3G - UMTS - } \\
\text { CDMA2000 -EDGE }\end{array}$ & Superior a 2 Mbps & Superior a $10 \mathrm{~km}$ & \multirow{3}{*}{$\begin{array}{l}\text { - NAN, WAN, } \\
\text { - DER, AMI, EV, ADA }\end{array}$} \\
\hline & Celular 3.5G & $14 \mathrm{Mbps}$ & Superior a $10 \mathrm{~km}$ & \\
\hline & Celular 4G-LTE & $\begin{array}{l}300 \text { Mbps y } 3.3 \text { Gbps para } \\
\text { LTE Avanzado }\end{array}$ & Superior a $10 \mathrm{~km}$ & \\
\hline & Internet Satelital & $1 \mathrm{Mbps}$ & 100 a $6000 \mathrm{~km}$ & WAN \\
\hline
\end{tabular}

Fuente: (Khan, et al., 2016; Routray y Sharmila, 2016; Mahmood, Javaid y Razzaq, 2015; Kuzlu, et al., 2014; Fang, et al., 2012; W. Wang, et al., 2011) 
los municipios, 1000 zonas WiFi públicas gratuitas a lo largo del territorio nacional, sostenibilidad de Puntos Vive Digital en estratos uno y dos y sostenibilidad de los 7621 Kioscos Vive Digital (Ministerio de Tecnologías de la Información y las Comunicaciones, 2016).

Asimismo, desde el marco de asignación eficiente del espectro, el Plan Vive Digital 2014-2018, propone: asignar mediante subasta $245 \mathrm{MHz}$ de espectro para banda ancha inalámbrica, actualizar la normativa para el uso eficiente del espectro, flexibilizar el uso del espectro, implementar el Primer Portal de Espectro Visible de la región y definir más espectro para más aplicaciones de uso "no licenciado". En este último caso y bajo el enfoque de la aplicación de las RI en Colombia, se tiene como objetivo fomentar el despliegue de nuevos tipos de interconexión, como loT y comunicaciones máquina a máquina (M2M; Ministerio de Tecnologías de la Información y las Comunicaciones, 2014).

\section{INTERNET DE LAS COSAS (IoT)}

Internet de las cosas, es una infraestructura global para la sociedad de información que ofrece servicios avanzados a la interconexión de los objetos físicos y virtuales, empleando las tecnologías interoperables de comunicación e información existentes y en evolución (International Telecommunication Union, 2012). El enfoque evolutivo de loT proporciona servicios inteligentes, fiables y seguros, cuyo impacto depende de la tecnología seleccionada, la capacidad organizacional y las políticas del lugar donde van a ser implementados (Mohanty, et al., 2016; Dalipi y Yayilgan, 2016). A partir de los requerimientos que ha generado el desarrollo de las RI, loT se ha convertido en la tecnología base para la comunicación bidireccional entre los actores de la red.

Debido al alto flujo de información en las RI, el procesamiento, la seguridad y privacidad son tres factores que se deben tener en cuenta para prevenir fallas o eventos que puedan comprometer la confidencialidad, integridad y disponibilidad de las TIC; por esta razón loT implementa tecnologías de procesamiento que gestionan gran cantidad de datos Big Data (BD, por sus siglas en inglés), a través de métodos algorítmicos de análisis avanzado y herramientas de recuperación de información útil (Mohanty, et al., 2016; Zeng, et al., 2016). Como caso particular, frente a la seguridad digital, el Consejo Nacional de Política Económica y Social (CONPES), en sus documentos Conpes 3701 y Conpes 3854, ha establecido las políticas de ciberseguridad, ciberdefensa, y gestión de riesgo en el entorno digital de Colombia (Planeación, 2011; Departamento Nacional de Planeación, 2016).

En concordancia con el Programa de Transformación Productiva (PTP) para potenciar el desarrollo económico de Colombia, el Ministerio de las TIC con apoyo de Colciencias y una alianza estratégica entre universidades, líderes tecnológicos mundiales y empresas, impulsó la iniciativa de creación del Centro de Excelencia y Apropiación en Internet de las Cosas (CEA-IoT). De esta manera el CEA-IoT busca posicionar a Colombia como líder regional en TIC, a través de la identificación e implementación de soluciones tecnológicas basadas en loT dentro de las siguientes líneas: salud, logística, industria, vestibles, seguridad, agroindustria, medio ambiente y gobierno orientado a la conformación de ciudades inteligentes.

\section{AGRADECIMIENTOS}

Los autores agradecen a todos los miembros del proyecto Centro de excelencia y apropiación en internet de las cosas (CEA-IoT). Al igual que a todas las instituciones que financian este trabajo: Ministerio de Tecnologías de la Información y las Comunicaciones (MinTIC), y Departamento Administrativo de Ciencia, Tecnología e Innovación, Colciencias a través del Fondo Nacional de Financiamiento para la Ciencia, la Tecnología y la Innovación Francisco José de Caldas (ID del proyecto: FP44842-502-2015). 


\section{CONCLUSIONES}

El gobierno colombiano desde el marco regulatorio de la Ley 1715 de 2014, ha promovido la inversión del sector público y privado en la investigación y desarrollo de proyectos que empleen principalmente las FNCER. Mediante planes de acción, orientados a la sostenibilidad ambiental, económica y política, se han definido propuestas basadas en energías eólica, solar, biomasa y geotérmica, con el propósito de facilitar el acceso de las ZNI al servicio de energía eléctrica, garantizar el acceso continuo a la energía independiente de fenómenos climáticos, reducir el riesgo en períodos de escasez y disminuir el tiempo total de interupción por cliente.

Por tanto, desde el contexto de la seguridad eléctrica, equidad social y mitigación del impacto ambiental, el sistema energético colombiano debe afrontar los nuevos retos, requeridos para satisfacer las necesidades de la demanda. Desde un punto de vista técnico, es necesario dotar la red tradicional de las características de una RI, que integre gradualmente las tecnologías DER, ADA, AMI y EV; desde una perspectiva legal es imprescindible generar un esquema normativo y regulatorio claro y objetivo que propenda por el desarrollo de este tipo de tecnología. De esta manera, es fundamental disponer de un modelo de referencia como SGAM, que permita de una forma flexible identificar y visualizar las funciones e interoperabilidad de los componentes técnicos, normativos y regulatorios requeridos en esta transición.

Las RI ofrecen a Colombia la oportunidad de actualizar la cadena de conversión de energía eléctrica; es necesario disponer de una red eléctrica con operaciones completamente automatizadas, transmisión de información en tiempo real, disposición de sistemas alternos para análisis y gestión inteligente, reconfiguración automática, detección de fallas con servicios de aislamiento y restauración, que permitan a través de las TIC y en particular de IoT, la integración de los usuarios como agentes de participación activos dentro del mercado energético.

\section{REFERENCIAS}

Asamblea Nacional Constituyente. Constitución Política de Colombia de 1991 (1991). Recuperado de http://doi.org/10.1017/CBO9781107415324.004

Braslavsky, J., Wall, J. and Reedman, J. (2015). Optimal distributed Energy Resources and the Cost of Reduced Greenhouse Gas Emissions in a Large Retail Shopping Centre. Applied Energy, 155, 120-130, doi: http://doi.org/10.1016/j.apenergy.2015.05.085

Budka, K., Deshpande, J. and Thottan, M. (2014). Communication Networks for Smart Grids. Swindon, UK: Springer, doi: http://doi. org/10.1007/978-1-4471-6302-2

Caicedo, E., Castillo, J., Morales, W., Echeverry, R. y García, J. (2015). Metodología para la evaluación de proyectos pilotos en "Smart Grid" en Colombia. Recuperado de http://www.upme.gov.co/ Estudios/2016/SmartGrids2030/4_Parte4_Anexo9_ Proyecto_SmartGrids.pdf

Carvajal, S. y David, M. (2013). Impacto de la generación distribuida en el sistema eléctrico de potencia colombiano: un enfoque dinámico. Revista Tecnura, 77-89. Recuperado de http://revistas. udistrital.edu.co/ojs/index.php/Tecnura/article/ view/6886/8479

CEN-CENELEC-ETSI Smart Grid Coordination Group. (2012). Smart Grid Reference Architecture, 1-107. Retrieved from ftp://ftp.cen.eu/EN/EuropeanStandardization/HotTopics/SmartGrids/Security.pdf

CEN-CENELEC-ETSI Smart Grid Coordination Group. (2014). SG-CG/M490/K_SGAM usage and examples, 1-67. Retrieved from ftp://ftp.cencenelec. eu/EN/EuropeanStandardization/HotTopics/SmartGrids/SGCG_Methodology_SGAMUserManual.pdf

Cespedes, R., León, R. A., Salazar, H., Ruiz, M. E., Hidalgo, R. and Mejía, D. (2012). An Appraisal of the Challenges and Opportunities for the Colombia Inteligente Program Implementation. IEEE POwer and Energy Society General Meeting, 1-6, doi: http:// doi.org/10.1109/PESGM.2012.6345383

Chen, N., Tan, C. W. and Quek, T. Q. S. (2014). Electric Vehicle Charging in Smart Grid: Optimality and Valley-Filling Algorithms. IEEE Journal on Selected 
Topics in Signal Processing, 8(6), 1073-1083, doi: http://doi.org/10.1109/JSTSP.2014.2334275

Comité Directivo Colombia Inteligente. (2014). Informe de Gestión 2013. Colombia Inteligente, Informe de Gestión 2013, 1-75. Recuperado de http://www. colombiainteligente.com.co/banco_informacion/ Presentaciones/Informe de Gestión Colombia Inteligente 2013 web.pdf

Congreso de la República de Colombia. (1994). Régimen Básico. Recuperado de http://www.superservicios.gov.co/content/download/4977/47233

Congreso de la República de Colombia. (2014). Ley 1715 del 13 de Mayo de 2014. Recuperado de http://www.upme.gov.co/Normatividad/Nacional/2014/LEY_1715_2014.pdf

Correa, C., Marulanda, G. y Panesso, A. (2016). Impacto de la penetración de la energía solar fotovoltaica en sistemas de distribución: estudio bajo supuestos del contexto colombiano. Revista Tecnura, 20(50), 85-95, doi: http://doi.org/10.14483/udistrital.jour. tecnura.2016.4.a06

Cruz, R., Torres, H., Montoya, M., Barrientos, J., Pineda, L., Niebles, L. y Franco, C. (2013). Caracterización del sector eléctrico Colombiano, 364. Recuperado de http://repositorio.sena.edu.co/bitstream/11404/2138/1/3058.pdf

Dalipi, F. and Yayilgan, S. (2016). Security and Privacy Considerations for IoT Application on Smart Grids: Survey and Research Challenges. 2016 IEEE 4th International Conference on Future Internet of Things and Cloud Workshops (FiCloudW), 63-68, doi: http://doi.org/10.1109/W-FiCloud.2016.28

Dangond, C., Jolly, J., Monteoliva, A. y Rojas, F. (2011). Algunas reflexiones sobre la movilidad urbana en Colombia desde la perspectiva del desarrollo humano. Papel Político, 16 (2)(2), 485-514. Recuperado de http://www.scielo.org.co/pdf/papel/v16n2/ v16n2a07.pdf

Departamento Nacional de Planeación. (2016). Conpes 3854-Política Nacional de Seguridad Digital. Recuperado de https://colaboracion.dnp.gov.co/CDT/ Conpes/Económicos/3854.pdf
Dimitrov, P., Piroddi, L. and Prandini, M. (2016). Distributed Allocation Of A Shared Energy Storage System in a Microgrid, 3551-3556.

Elkhatib, M., Shatshat, R. and Salama, M. (2012). Decentralized Reactive Power Control for Advanced Distribution Automation Systems. IEEE Transactions on Smart Grid, 3(3), 1482-1490, doi: http://doi. org/10.1109/TSG.2012.2197833

Emmanuel, M. and Rayudu, R. (2016). Communication Technologies For Smart Grid Applications: A Survey. Journal of Network and Computer Applications, 74, 133-148. http://doi.org/10.1016/j. jnca.2016.08.012

Erol-Kantarci, M. and Mouftah, H. (2015). Energy-Efficient Information and Communication Infrastructures in the Smart Grid: A Survey on Interactions and Open Issues. IEEE Communications Surveys and Tutorials, 17(1), 179-197, doi: http://doi.org/10.1109/ COMST.2014.2341600

Fang, X., Misra, S., Xue, G. and Yang, D. (2012). Smart Grid-The New and Improved Power Grid: A Survey. IEEE Communications Surveys and Tutorials, 14(4), 944-980, doi: http://doi.org/10.1109/ SURV.2011.101911.00087

Georgilakis, P., Member, S. and Hatziargyriou, N. D. (2013). Optimal Distributed Generation Placement in Power Distribution Networks: Models, Methods and Future Research. IEEE Transactions on Power Systems, 28(3), 3420-3428, doi: http://doi. org/10.1109/TPWRS.2012.2237043

Gopi, C. and Lalu, V. (2016). Sensor Network Infrastructure for AMI in Smart Grid. Procedia Technology, 24, 854-863, doi: http://doi.org/10.1016/j. protcy.2016.05.133

Grupo Técnico Proyecto BID Marco de Cooperación Técnica CO-T1337. (2016a). Smart Grids Colombia Visión 2030 Parte I. Recuperado de http://www. upme.gov.co/Estudios/2016/SmartGrids2030/1_ Parte1_Proyecto_BID_Smart_Grids.pdf

Grupo Técnico Proyecto BID Marco de Cooperación Técnica CO-T1337. (2016b). Smart Grids Colombia Visión 2030 Parte II. Recuperado de http://www. upme.gov.co/Estudios/2016/SmartGrids2030/2_ Parte2_Proyecto_BID_Smart_Grids.pdf 
Grupo Técnico Proyecto BID Marco de Cooperación Técnica CO-T1337. (2016c). Smart Grids Colombia Visión 2030 Parte IV Anexo 6. Estimación de Beneficios detallados por Tecnología. Recuperado de http://www.upme.gov.co/Estudios/2016/SmartGrids2030/4_Parte4_Anexo6_Proyecto_SmartGrids.pdf

Guerrero, J., Chandorkar, M., Lee, T. y Loh, P. (2013). Advanced Control Architectures for Intelligent Microgrids; Part I: Decentralized and Hierarchical Control. Industrial Electronics, IEEE Transactions on, 60(4), 1254-1262, doi: http://doi.org/10.1109/ TIE.2012.2194969

Guerrero, J., Loh, P., Lee, T. and Chandorkar, M. (2013). Advanced Control Architectures for Intelligent $\mathrm{Mi}$ crogrids Part II: Power quality, energy storage, and AC/DC microgrids. IEEE Transactions on Industrial Electronics, 60(4), 1263-1270, doi: http://doi. org/10.1109/TIE.2012.2196889

Ho, Quang-Dung, Chao, Chon-Wang, Derakhshani, Mahsa, Le-Ngoc, T. (2014). An Analysis on Throughput and Feasibility of Narrow-band Power Line Communications in Advanced Distribution Automation Scenarios, 4233-4238.

International Energy Agency. (2011). Technology Roadmap-Smart Grids. Current, 52, doi: http://doi. org/10.1007/SpringerReference_7300

International Telecommunication Union. Overview of the Internet of things, ITU-T 22 (2012). Retrieved from http://www.itu.int/ITU-T/recommendations/ rec.aspx? rec $=11559$ \&lang $=\mathrm{es}$

ITU-T Focus Group on Smart Sustainable Cities. (2014). Una visión general de las ciudades inteligentes sostenibles y el papel de las tecnologías de la información y comunicación.

ITU-T Focus Group on Smart Sustainable Cities. (2015). Standardization Roadmap for Smart Sustainable Cities.

Justo, J., Mwasilu, F., Lee, J. and Jung, J. (2013). AC-microgrids Versus DC-Microgrids with Distributed Energy Resources: A Review. Renewable and Sustainable Energy Reviews, 24, 387-405, doi: http:// doi.org/10.1016/j.rser.2013.03.067
Kennel, F., Gorges, D. and Liu, S. (2013). Energy Management for Smart Grids With Electric Vehicles Based on Hierarchical MPC. Industrial Informatics, IEEE Transactions on, 9(3), 1528-1537, doi: http:// doi.org/10.1109/TII.2012.2228876

Khan, A., Rehmani, M. and Reisslein, M. (2016). Cognitive Radio for Smart Grids: Survey of Architectures. Spectrum Sensing Mechanisms and Networking Protocols, 18(1), 860-898.

Kuzlu, M., Pipattanasomporn, M. and Rahman, S. (2014). Communication Network Requirements for Major Smart Grid Applications in HAN, NAN and WAN. Computer Networks, 67, 74-88, doi: http:// doi.org/10.1016/j.comnet.2014.03.029

Lee, G. y Su, D. (2013). Standarization of Smart Grid in ITU-T. IEEE Communications Magazine, 75-83. Retrieved from http://ieeexplore.ieee.org/xpls/abs_all. jsp?arnumber $=6400442$

Li, M., Xiao, H., Gao, W. and Li, L. (2016). Smart Grid Supports the Future Intelligent City Development, 6128-6131.

Li, W. and Zhang, X. (2014). Simulation of the Smart Grid Communications: Challenges, Techniques, and Future Trends. Computers and Electrical Engineering, 40(1), 270-288, doi: http://doi.org/10.1016/j.compeleceng.2013.11.022

Madani, V., Das, R., Aminifar, F., McDonald, J., Venkata, S. S., Novosel, D. and Shahidehpour, M. (2015). Distribution Automation Strategies Challenges and Opportunities in a Changing Landscape. IEEE Transactions on Smart Grid, 6(4), 2157-2165, doi: http://doi.org/10.1109/TSG.2014.2368382

Mahmood, A., Javaid, N. and Razzaq, S. (2015). A Review of Wireless Communications for Smart Grid. Renewable And Sustainable Energy Reviews, 41, 248-260, doi: http://doi.org/10.1016/j. rser.2014.08.036

Marzband, M., Parhizi, N., Savaghebi, M. and Guerrero, J. (2016). Distributed Smart Decision-Making for a Multimicrogrid System Based on a Hierarchical Interactive. Architecture. IEEE Transactions on Energy Conversion, 31(2), 637-648.

Ministerio de Comercio Industria y Turismo. (2014). Programa de Transformación Productiva. Informe 
de Gestión 2014. Recuperado de https://www. ptp.com.co/documentos/informe de gestion 2014 PTP-.pdf

Ministerio de Minas y Energía. (2015a). Decreto 1073 de mayo 26 de 2015. Recuperado de https://www.minminas.gov.co/ documents/10180/170046/Decreto+\%F2ni$\mathrm{CO}+$ Reglamentario+Sector+Minas+y+Energ\%92a. pdf/8f19ed1d-16a0-4a09-8213-ae612e424392

Ministerio de Minas y Energía. (2015b). Decreto 2143 de noviembre 4 de 2015. Recuperado de http:// wp.presidencia.gov.co/sitios/normativa/decretos/2015/Decretos2015/DECRETO 2143 DEL 04 DE NOVIEMBRE DE 2015.pdf

Ministerio de Tecnologías de la Información y las Comunicaciones. (2014). Plan Vive Digital 20142018. Recuperado de http://www.mintic.gov.co/ portal/604/articles-5193_recurso_2.pdf

Ministerio de Tecnologías de la Información y las Comunicaciones. (2016). Resolución 1564 de agosto 25 de 2016. Recuperado de http://www.mintic.gov. co/portal/604/articles-16903_documento.pdf

Mohanty, B., Choppali, U. and Kougianos, E. (2016). Everything you Wanted to Know About Smart Cities. IEEE Consumer Electronics Magazine, 60-70, doi: http://doi.org/10.1109/MCE.2016.2556879

Monteiro, V., Pinto, J. and Afonso, J. (2016). Operation Modes for the Electric Vehicle in Smart Grids and Smart Homes: Present and Proposed Modes. IEEE Transactions on Vehicular Technology, 65(3), 1007-1020, doi: http://doi.org/10.1109/ TVT.2015.2481005

Montes, C., Slootweg, H. and Van Eekelen, M. (2013). Structuring the Design Space of the ICT Architecture for the Smart Grid. IEEE Recent Advances in Intelligent Computational Systems, RAICS 2013, 110-115, doi: http://doi.org/10.1109/ RAICS.2013.6745456

Mukherjee, J. and Gupta, A. (2015). A Review of Charge Scheduling of Electric Vehicles in Smart Grid. IEEE Systems Journal, 9(4), 1541-1553, doi: http://doi. org/10.1109/JSYST.2014.2356559
Neureiter, C., Uslar, M., Engel, D. and Lastro, G. (n.d.). A Standards-based Approach for Domain Specific Modelling of Smart Grid System Architectures.

Nieße, A., Tröschel, M. and Sonnenschein, M. (2014). Designing Dependable and sustainable Smart Grids-How to apply Algorithm Engineering to distributed control in power systems. Environmental Modelling and Software, 56, 37-51, doi: http://doi. org/10.1016/j.envsoft.2013.12.003

Ozgur, U., Tonyali, S., Akkaya, K. and Senel, F. (2016). Comparative Evaluation of Smart Grid AMI Networks. Performance under Privacy, 1-3.

Planeación, D. N. de. (2011). Documento Conpes 3701 de julio 14 de 2011. Recuperado de http://www. mintic.gov.co/index.php/docs-normatividad?task=download. . ile \&fid $=46.741 \&$ sid $=54$

Quintana, B. (2014). Modelo de masificación de vehículos eléctricos en Bogotá D.C. Bogotá, D.C.: Universidad Nacional de Colombia. Departamento De Ingeniería Eléctrica Y Electrónica. Recuperado de http://www.bdigital.unal.edu. co/48580/1/73575424.2015.pdf

Rahman, H., Majid, M., Rezaee, A., Chin Kim, G., Hassan, M. and Fadhl, S. (2015). Operation and Control Strategies of Integrated Distributed Energy Resources: A Review. Renewable and Sustainable Energy Reviews, 51, 1412-1420, doi: http://doi.org/10.1016/j.rser.2015.07.055

Rashed, R., Fung, A., Mohammadi, F. and Raahemifar, K. (2014). A survey on Advanced Metering Infrastructure. International Journal of Electrical Power and Energy Systems, 63, 473-484, doi: http://doi. org/10.1016/j.ijepes.2014.06.025

Routray, S. and Sharmila, K. (2016). 4.5G : A Milestone Along the Road to 5C.

UPME. (2013). Plan indicativo de expansión de cobertura de energía eléctrica 2013-2017, doi: http://doi. org/10.1017/CBO9781107415324.004

UPME. (2015a). Plan de Expansión de Referencia Generación-Transmisión 2015-2029. Recuperado de http:// www.upme.gov.co/Docs/Plan_Expansion/2016/ Plan_Expansion_GT_2015-2029/Plan_GT_20152029_VF_22-12-2015.pdf 
UPME. (2015b). Plan Energético Nacional Colombia: Ideario Energético 2050. Unidad de Planeación Minero Energética. Recuperado de http://www.upme. gov.co/Docs/PEN/PEN_IdearioEnergetico2050.pdf

UPME. (2016). Plan de Expansión de Referencia Generación-Transmisión 2016-2030. Recuperado de http:// www.upme.gov.co/Docs/Plan_Expansion/2016/ Plan_GT_2016_2030/Plan_GT_2016_2030_Final_ V1_12-12-2016.pdf

UPME y BID. (2015). Integración de las energías renovables no convencionales en Colombia. Recuperado de http://www.upme.gov.co/Estudios/2015/Integracion_Energias_Renovables/INTEGRACION_ENERGIAS_RENOVANLES_WEB.pdf

Vasquez, J., Guerrero, J. M., Miret, J., Castilla, M. and Vicuña, L. (2010). Hierarchical Control of Intelligent Microgrids. IEEE Industrial Electronics Magazine, 23-29, doi: http://doi.org/10.1109/ MIE.2010.938720

Veldman, E. and Verzijlbergh, R. (2015). Distribution Grid Impacts of Smart Electric Vehicle Charging from Different Perspectives. IEEE Transactions on Smart Grid, 6(1), 333-342, doi: http://doi. org/10.1109/TSG.2014.2355494

Wang, W., Xu, Y. and Khanna, M. (2011). A Survey on the Communication Architectures In Smart Grid. Computer Networks, 55, 3604-3629, doi: http:// doi.org/10.1016/j.comnet.2011.07.010

Wang, X., Guerrero, J. , Chen, Z. and Blaabjerg, F. (2010). Distributed Energy Resources in Grid
Interactive AC Microgrids. 2nd International Symposium on Power Electronics for Distributed Generation Systems, PEDG 2010, 806-812, doi: http:// doi.org/10.1109/PEDG.2010.5545802

Wei, X., Zhou, Y. and Zhu, J. (2009). Energy-efficient Distribution in Smart Grid. 1st International Conference on Sustainable Power Generation and Supply, SUPERGEN '09, 1-6, doi: http://doi.org/10.1109/ SUPERGEN.2009.5348223

World Energy Council. (2015). 2015 Energy Trilemma Index-Benchmarking the Sustainability of National Energy Systems, 306. Retrieved from https://www.worldenergy.org/wp-content/ uploads/2015/11/20151030-Index-report-PDF.pdf

Xing, Y., Jin, J., Wang, Y., Du, B., Wang, S. and Modeling, A. (2016). An Electric Vehicle Charging System Using an SMES Implanted Smart Grid, 26(7).

Yu, K., Zhu, L., Wen, Z., Mohammad, A., Zhou, Z. and Sato, T. (2014). CCN-AMI : Performance Evaluation of Content- Centric Networking Approach for Advanced Metering Infrastructure in Smart Grid.

Zavoda, F. (2010). Advanced distribution automation (ADA) applications and Power Quality in Smart Grids. CICED 2010 Proceedings, (Vvc), 1-7.

Zeng, X., Garg, S., Strazdins, P., Jayaraman, P., Georgakopoulos, D. and Ranjan, R. (2016). IOTSim: A simulator for analysing loT applications. Journal of Systems Architecture, 0, 1-15, doi: http://doi.org/10.1016/j.sysarc.2016.06.008

\section{(c) $(1) \Theta \Theta$}

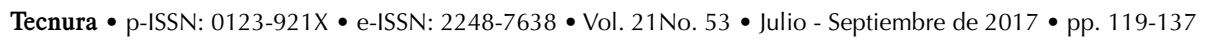

